\title{
The production of charm mesons from quark matter at CERN SPS and RHIC
}

\author{
P. Lévai, T.S. Biró, P. Csizmadia, T. Csörgö and J. Zimányi \\ KFKI Research Institute for Particle and Nuclear Physics, \\ P. O. Box 49, Budapest, 1525, Hungary
}

\begin{abstract}
We study the production of charm mesons and other charm baryons from quark matter at CERN SPS and RHIC energies. Using quark coalescence models as hadronization mechanism, we predict particle ratios, absolute yields and transverse momentum spectra.

PACS numbers: 12.38.Mh, 13.87.Fh, 24.85.+p
\end{abstract}

\section{Introduction}

During the last decade $J / \psi$ production was investigated with great interest at the CERN Super Proton Synchrotron (SPS), and an anomalous suppression has been detected in $\mathrm{Pb}+\mathrm{Pb}$ collisions at $158 \mathrm{GeV} /$ nucleon bombarding energy [i]. This result is is assumed to be the strongest evidence of QGP formation (for an overview see Ref. [2]). However, the unambiguous confirmation of the QGP formation requires more charm related data, e.g. absolute yields, transverse momentum spectra and the characteristics of charm hadron production from deconfined matter.

In this paper we summarize our results obtained by assuming quark coalescence as microscopical hadronization mechanism of the possibly produced deconfined phase at CERN SPS energy. The ALCOR [3, 6, 5] and MICOR [6, 7] hadronization models were extended by including charm quark and the consecutive charm hadron formation.

\section{The number of charm quarks in heavy ion collision at SPS energy}

Perturbative QCD calculations predict small number of newly produced charm quarkantiquark pairs in the $\mathrm{Pb}+\mathrm{Pb}$ collision at CERN SPS energy, $N_{c \bar{c}} \approx 0.1$. On the other hand statistical models obtain a much larger number, $N_{c \bar{c}} \approx 8.5$ [8]. If we assume that deconfinement appears after the formation of a hot dense string matter where strings become ropes in the presence of large density (characterized by an increased string constant, $\kappa)$, then quark-antiquark pair creation will be dominated by the breaking of these in-medium strings/ropes. The enhancement of the heavy quark-antiquark production, $\gamma_{X}$, can be calculated by means of the Schwinger mechanism [9]:

$$
\gamma_{X}=\frac{P(X \bar{X})}{P(q \bar{q})}=\exp \left(-\frac{\pi\left(m_{X}^{2}-m_{q}^{2}\right)}{\kappa}\right)
$$

If we increase the usual value of the string constant $\kappa=1.0 \mathrm{GeV} / \mathrm{fm}$ with $10 \%$, then an enhancement factor 24 appears for charm quarks with mass $m_{c}=1.5 \mathrm{GeV}$ $\left(m_{q}=223 \mathrm{MeV}\right) .20 \%$ increase in $\kappa$ would generate an enhancement factor 350 !!! 
Thus relatively small change in the string breaking could modify dramatically the total charm production in heavy ion collisions. If relatively large number of charm quarks can be produced in the initial phase of the heavy ion collision via in-medium breaking of the primary strings, then after deconfinement the quark coalescence can become a reliable description of charm hadron formation from the plasma state.

\section{Quark coalescence}

In our description quark coalescence is the basic hadronization mechanism. It is assumed that the number of directly produced hadrons is given by the product of the number of quarks (or anti-quarks) from which those hadrons are produced, multiplied by coalescence coefficients $C_{h}$ and by non-linear normalization coefficients $b_{q}$, that take into account conservation of quark numbers during quark coalescence [3, 14. The number of various quarks is denoted by the symbol usual for that type of particles, e.q. $u, d, s$ and $c$ denote the number of light, strange and charmed quarks, respectively. The following examples display the appropriate coalescence relations (see Ref. [5]):

$$
\begin{array}{rlrl}
p^{+}(u u d) & =C_{p} \cdot\left(b_{u} u\right)^{2} \cdot\left(b_{d} d\right) & K^{+}(u \bar{s}) & =C_{K} \cdot\left(b_{u} u\right) \cdot\left(b_{\bar{s}} \bar{s}\right) \\
& \ldots & & \ldots \\
\Omega(s s s) & =C_{\Omega} \cdot\left(b_{s} s\right)^{3} & \phi(s \bar{s}) & =C_{\phi} \cdot\left(b_{s} s\right) \cdot\left(b_{\bar{s}} \bar{s}\right) \\
\Omega_{c}(s s c) & =C_{\Omega}^{c} \cdot\left(b_{s} s\right)^{2} \cdot\left(b_{c} c\right) & D^{+}(c \bar{d}) & =C_{D} \cdot\left(b_{c} c\right) \cdot\left(b_{\bar{d}} \bar{d}\right) \\
\Omega_{c c}(s c c) & =C_{\Omega}^{c c} \cdot\left(b_{s} s\right) \cdot\left(b_{c} c\right)^{2} & D_{s}^{+}(c \bar{s}) & =C_{D}^{s} \cdot\left(b_{c} c\right) \cdot\left(b_{\bar{s}} \bar{s}\right) \\
\Omega_{c c c}(c c c) & =C_{\Omega}^{c c c} \cdot\left(b_{c} c\right)^{3} & J / \psi(c \bar{c}) & =C_{J / \psi} \cdot\left(b_{c} c\right) \cdot\left(b_{\bar{c}} \bar{c}\right)
\end{array}
$$

The non-linear coalescence factors $b_{u}, b_{d}, b_{s}, b_{c}$ and the $b_{\bar{u}}, b_{\bar{d}}, b_{\bar{s}}, b_{\bar{c}}$ are determined unambiguously from the requirement that the number of constituent quarks and antiquarks do not change during the hadronization, and that all initially available quarks and anti-quarks have to end up in the directly produced hadrons. This constraint is a basic assumption in all models of quark coalescence. The correct quark counting yields to 8 coupled non-linear equations, expressing the conservation of the number of quarks and antiquarks. Without solving these non-linear equations, one can recognize interesting relations among the different hadron ratios. For example certain meson ratios play the role of step functions among different antibaryon to baryon ratios. This is illustrated in Figure 1 from Ref. [5], where the $u$ and $d$ quarks are considered together as $q$ light quark.

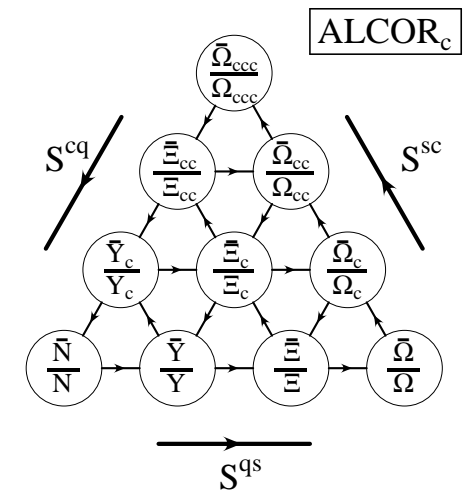

Figure 1. Mesonic step factors $\mathcal{S}^{q s}, \mathcal{S}^{s c}, \mathcal{S}^{c q}$ and antibaryon to baryon ratios. 
Here the step factors are defined as:

$$
\begin{aligned}
& \mathcal{S}^{q s} \equiv \frac{K(q \bar{s})}{\bar{K}(\bar{q})}=\left[\frac{b_{q} q}{b_{\bar{q}} \bar{q}}\right] \cdot\left[\frac{b_{\bar{s}} \bar{s}}{b_{s} s}\right], \\
& \mathcal{S}^{s c} \equiv \frac{\bar{D}_{s}(s \bar{c})}{D_{s}(\bar{s} c)}=\left[\frac{b_{s} s}{b_{\bar{s}} \bar{s}}\right] \cdot\left[\frac{b_{\bar{c}} \bar{c}}{b_{c} c}\right], \\
& \mathcal{S}^{c q} \equiv \frac{D(\bar{q} c)}{\bar{D}(q \bar{c})}=\left[\frac{b_{c} c}{b_{\bar{c}} \bar{c}}\right] \cdot\left[\frac{b_{\bar{q}} \bar{q}}{b_{q} q}\right] .
\end{aligned}
$$

These properties lead to the identity $\mathcal{S}^{q s} \cdot \mathcal{S}^{s c} \cdot \mathcal{S}^{c q} \equiv 1$, which can be rewritten as a measurable relation between these mesonic ratios: $\left(\bar{D}_{s} / D_{s}\right) /(\bar{D} / D)=\bar{K} / K$. These relations are valid at both CERN SPS and RHIC energies, if quark coalescence describes the hadronization of the produced deconfined phase.

\section{Predictions for charm hadron yields at SPS and RHIC}

Total hadron yields can be determined through the numerical solution of the appropriate 8 non-linear equations of quark number conservation. The input values are the newly produced quark-antiquark numbers, $N_{u \bar{u}}, N_{d \bar{d}}, N_{s \bar{s}}$ and $N_{c \bar{c}}$. At CERN SPS energy the earlier ALCOR calculations could successfully reproduce most of the

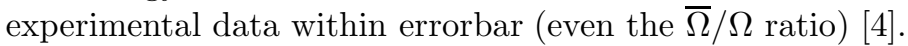

Including the charm quark into this model, one experimental data about charm hadron production is needed to determine the full charm production yield. We choose the known ratio $J / \psi / h^{-}=1.0 \cdot 10^{-6}[8]$. The ALCOR model can reproduce this data with $N_{c \bar{c}}=3.4$ 10]. The obtained charm hadron yields (see Table 1) display a factor of 12 enhancement relative to the perturbative QCD predictions, but they reach only $40 \%$ of the predictions from the statistical model [8].

We repeat our calculation at RHIC energies (see Table 1), assuming that the relative strange and charm quark-antiquark pair production remains the same, only the absolute yields are scaled with increasing energy, following the increase in the number of $h^{-}$( $h^{-}$was obtained from the HIJING model). Table 1 shows that detectable numbers of charm hadrons $\left(D, \Lambda_{c}, \bar{\Lambda}_{c}\right)$ ought to be produced at RHIC.

\section{Transverse momentum spectra of the $\mathbf{D}$ and $J / \psi$ mesons}

The transverse momentum spectra of the produced hadrons can be calculated by the MICOR model [6, 7]. Applying this model one can determine the hadronization temperature $T_{H A D}$ and the transverse flow of the hadronizing quarks. At CERN SPS energy $T_{H A D}=175 \mathrm{MeV}$ and $v_{T}=0.46 \pm 0.05$ fit the data in the $\mathrm{Pb}+\mathrm{Pb}$ collision [6]. Including charm quarks into this model we can determine the transverse momentum spectra of the $D$ and the $J / \psi$ mesons. Figure 2 displays the obtained

values $T_{\text {eff }}^{D}=259 \pm 19 \mathrm{MeV}$ and $T_{e f f}^{J / \psi}=315 \pm 35 \mathrm{MeV}$ for the slope parameter of the transverse momentum spectra [7]. We urge future experiments to measure these quantities at CERN SPS.

\section{Acknowledgments}

This work was supported by the Hungarian OTKA Grant T024094, T025579, and partly by the US-Hungarian Joint Fund No. 652. 
The production of charm mesons from quark matter at CERN SPS and RHIC

Table 1. Charm hadron production at CERN SPS and RHIC energies from the ALCOR model

\begin{tabular}{llll}
\hline & $\sqrt{s}=17.4 \mathrm{~A} \mathrm{GeV}$ & $\sqrt{s}=56 \mathrm{~A} \mathrm{GeV}$ & $\sqrt{s}=130 \mathrm{~A} \mathrm{GeV}$ \\
\hline$h^{-}$ & 680 & 1131 & 1828 \\
\hline$N_{c \bar{c}}$ & 3.4 & 6.7 & 12 \\
$\left\langle D^{0}+\bar{D}^{0}\right\rangle$ & 2.4 & 4.2 & 6.4 \\
$\langle J / \psi\rangle / h^{-}$ & $1.0 \cdot 10^{-6}$ & $1.5 \cdot 10^{-6}$ & $1.6 \cdot 10^{-6}$ \\
$\langle J / \psi\rangle /\left\langle D^{0}+\bar{D}^{0}\right\rangle$ & $3.0 \cdot 10^{-4}$ & $4.1 \cdot 10^{-4}$ & $4.6 \cdot 10^{-4}$ \\
$\Lambda_{c}$ & 2.2 & 3.7 & 6.1 \\
$\bar{\Lambda}_{c}$ & 0.3 & 1.7 & 4.0 \\
\hline
\end{tabular}

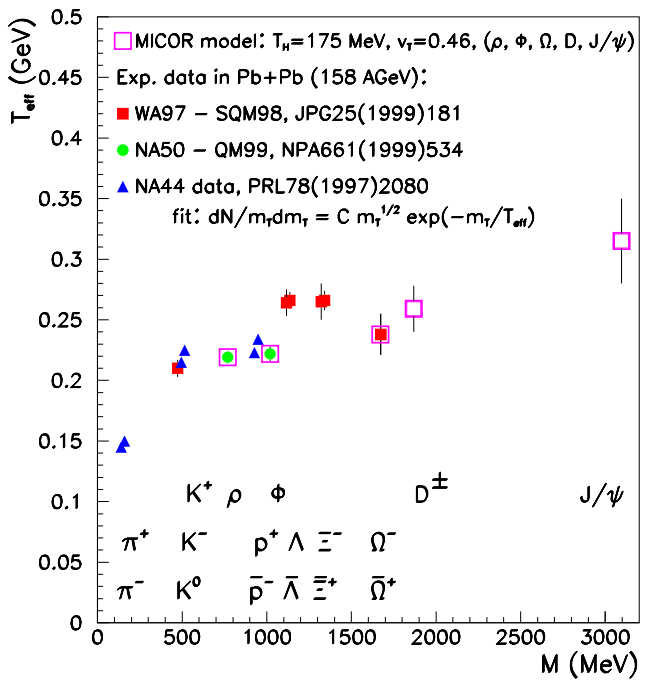

Figure 2. Experimental hadronic slopes of the transverse momentum spectra in the $\mathrm{PbPb}$ collision at $158 \mathrm{AGeV}$ energy from WA97 (squares), NA50 (dots) and NA44 Collaborations (triangulars), see Ref. [7]. Open squares with errorbar indicate the MICOR results on $D$ and $J / \psi$ mesons.

\section{References}

[1] Abreau M C et al., NA50 Coll., 1999 Phys. Lett. B 450 456; 2000 Phys. Lett. B 47728.

[2] Satz H 2000 Preprint hep-ph/0007069.

[3] Biró T S, Lévai P and Zimányi J 1995 Phys. Lett. B 347 6; 1997 J. Phys. G: Nucl. Part. Phys. 23 1941; 1999 J. Phys. G: Nucl. Part. Phys. 23311.

[4] Zimányi J, Biró T S and Lévai P 1999 Phys. Rev. C 591574.

[5] Lévai P, Biró T S, Csörgő T and Zimányi J 2000 Preprint hep-ph/0007247

[6] Csizmadia P et al. 1999 J. Phys. G: Nucl. Part. Phys. 23 321; Csizmadia P and Lévai P 2000 Phys. Rev. C 61031903.

[7] Csizmadia P and Lévai P 2000 Preprint hep-ph/0008195

[8] Gaździcki M and Gorenstein M I 1999 Phys. Rev. Lett. 83 4009; 1999 Acta Phys. Polon. B 30 2705; Gaździcki M and Markert C 2000 Acta Phys. Polon. B 31965.

[9] Bleicher M, Greiner W, Stöcker H, Xu N 2000 Preprint hep-ph/0007215.

[10] Lévai P, Biró T S, Zimányi J 2000 in preparation. 